\title{
A Molecular Dynamics Study of Thermodynamic and Kinetic Properties of Solid-Liquid Interface for Bcc Iron
}

\author{
Yusuke WATANABE, Yasushi SHIBUTA and Toshio SUZUKI \\ Department of Materials Engineering, The University of Tokyo, 7-3-1 Hongo, Bunkyo-ku, Tokyo 113-8656 Japan. E-mail: \\ shibuta@material.t.u-tokyo.ac.jp
}

(Received on April 1, 2010; accepted on May 28, 2010)

\begin{abstract}
Molecular dynamics simulations have been performed to give an estimate on the solid-liquid interfacial properties of bcc iron, namely the kinetic coefficients and solid-liquid interfacial energy. The kinetic coefficients for different orientations were estimated from the propagation velocity of planar solid-liquid interfaces. The anisotropy of kinetic coefficients, $\mu$, was confirmed to be $\mu_{(100)}>\mu_{(110)}$, which is similar to the literatures using other interatomic potentials. Moreover, growing and shrinking behavior of the freestanding spherical crystal and semi-spherical crystal on the substrate in the undercooled liquid was examined. There is a critical temperature dividing shrink or growth of both the freestanding spherical crystal and semi-spherical crystal on the substrate. The solid-liquid interfacial energy was then estimated from Gibbs-Thomson relation in the critical temperature as a function of the inverse of crystal radius.
\end{abstract}

KEY WORDS: molecular dynamics simulation; solidification; solid-liquid interface; interfacial energy; kinetic coefficient.

\section{Introduction}

The thermodynamic and kinetic properties of the solid-liquid interface, namely kinetic coefficients and solid-liquid interfacial energy, are known to be important quantities governing the nucleation rates, and ultimately morphologies during solidification from undercooled melts. ${ }^{1)}$ Despite their importance, experimental procedures to measure such quantities are difficult because the interface lies between two dense phases of solid and liquid. ${ }^{2)}$ The kinetic coefficients have been measured for only limited materials experimentally ${ }^{3,4)}$ and relying heavily on computer simulations. For the estimation of solid-liquid interfacial energies, Turnbull ${ }^{5}$ measured nucleation rates at temperatures around the maximum undercooling for various materials. The relationships were compared with classical homogeneous nucleation theory, in which a nucleus for solidification generates from liquid. But even with the experimental technique of using small droplets to minimize impurities in melt, $\left.{ }^{6}\right)$ heterogeneous nucleation still play a dominant role in nucleation process. The other method is utilized for eutectic alloys and known to be more accurate: by observing the shape of grain boundary cusps. ${ }^{7}$ However, the number of experiments reported for this method is limited, with mostly aluminum alloys ${ }^{7)}$ and few other metals. ${ }^{7,8)}$

In order to overcome difficulties for measuring those properties, molecular dynamic (MD) simulations have contributed to the understanding of the atomistic behavior of the solid-liquid interface. Kinetic coefficients were estimated by using various interatomic potentials, with reports including the Lennard-Jones potential, ${ }^{9,10}$ hard-sphere model, ${ }^{11)}$ and embedded atom method (EAM). ${ }^{12,13)}$ Although absolute values of kinetic coefficients are unreliable since the melting temperatures do not match with the experimental values most of the time, general conclusions could be drawn that anisotropy in the growth behavior was the fastest for the (100) plane, compared to the (110) and (111) planes for both bcc and fcc metals. The solid-liquid interfacial energies has been estimated widely by molecular dynamics simulations, such as the cleaving technique ${ }^{14-16)}$ applying an external cleaving potential to estimate the anisotropic solid-liquid interfacial energy, an capillary fluctuation method, ${ }^{13,17,18)}$ in which the interfacial stiffness was extracted from the fluctuation of the solid-liquid interface profile, and estimation of the solid-liquid interfacial energy from Gibbs-Thomson coefficient in relation between critical nucleus sizes and the inverse of the critical undercooling temperature by treating spherical crystal. ${ }^{19-21)}$ However, there are some technical difficulties in these methods. For example, a lot of efforts are needed to deal with the stable interface in the cleaving technique. In addition, it is difficult to identify the solid and liquid atoms in the capillary fluctuation method to estimate interfacial stiffness, which depends on the criterion of one's choice strongly. Therefore, it is important to establish a reliable technique for the estimation of the solid-liquid interface. The technical aspect of the estimation method of the solid-liquid interface is summarized in Ref. 20).

Regarding the iron and steel, there are few established references on thermodynamic and kinetic properties of the solid-liquid interface estimated by MD simulation since there is no reliable interatomic potential for iron atoms. We 
have employed the Finnis-Sinclair (FS) potential ${ }^{22)}$ consistently to examine the structure and thermal properties of bec metals under various conditions ${ }^{23-27)}$ since FS potential can reproduce mechanical properties of the bcc metals correctly despite its simple form and short cutoff distance. In this study, based on previous studies, the kinetic coefficients and solid-liquid interfacial energy was estimated by MD simulation. First, planar solid-liquid interfaces were induced to calculate kinetic coefficients for (100) and (110) planes of bcc iron. Then, critical temperature dividing the growth or melting of freestanding spherical crystals in the undercooled liquid or semi-spherical crystals supported on the substrate contacting the undercooled liquid was examined in order to estimate the solid-liquid interfacial energy from the correlation between the depression of the critical temperature and the crystal size.

\section{Simulation Methodology}

The FS potential ${ }^{22)}$ was applied to describe the interactions between iron atoms. The total energy of the FS potential, $E$, is expressed in following equations:

$$
\begin{gathered}
E=\frac{1}{2} \sum_{i} \sum_{j} V_{i j}\left(r_{i j}\right)-A \sum_{i} \sqrt{\rho_{i}} \ldots \ldots \ldots \ldots \\
V_{i j}=\left\{\begin{array}{cc}
\left(r_{i j}-c\right)^{2}\left(c_{0}+c_{1} r_{i j}+c_{2} r_{i j}^{2}\right) & \left(r_{i j} \leq c\right) \\
0 & \left(r_{i j}>c\right)
\end{array}\right. \\
\rho_{i}=\sum_{j \neq i} \phi\left(r_{i j}\right) \ldots \ldots \ldots \ldots \ldots \ldots \ldots \ldots \\
\phi\left(r_{i j}\right)=\left\{\begin{array}{cc}
\left(r_{i j}-d\right)^{2}+\beta \frac{\left(r_{i j}-d\right)^{3}}{d} & \left(r_{i j} \leq d\right) \\
0 & \left(r_{i j}>d\right)
\end{array}\right.
\end{gathered}
$$

where $V$ represents the repulsive term, and $A$ represents the binding energy. $r_{i j}$ is the bond length between atoms $i$ and $j$, $\rho$ is the total electronic charge density at the site of atom $i$, which is constructed by the rigid superposition of atomic charge densities $\Phi$. The parameters taken from original works by Finnis and Sinclair ${ }^{22,28)}$ are listed in Table 1. Note that the melting point of iron for the bulk system under the FS potential has been estimated to be $2400 \pm 10 \mathrm{~K},{ }^{29)}$ about $600 \mathrm{~K}$ higher than the experimental value of $1808 \mathrm{~K}$.

The classical equation of motion was integrated with a leapfrog method with a time step of $5.0 \mathrm{fs}$. The Berendsen thermostat $^{30)}$ was applied to control the temperature for every step. The Andersen method ${ }^{31)}$ was applied for controlling pressure. The pressure in each direction was independently controlled using a piston with a mass of $1.0 \times$ $10^{-6} \mathrm{~kg}$. The periodic boundary conditions were applied except for the top and bottom boundary for calculation of the semi-spherical nucleus on the substrate in Sec. 3.3, where the mirror boundary was employed. For the visual clarification, the metal atoms with bcc configuration were defined by the numbers of atoms within two cutoff lengths. Specifically, the atoms satisfying the following conditions were identified as having a bcc configuration, ${ }^{24)}$ which is technically equivalent to solid atoms in this study: (i) has 8 atoms within the first cutoff distance, (ii) has 14 atoms in the coordinates between the first cutoff length and the second cutoff length. The two cutoff lengths were set to be 2.80 and $3.40 \AA$, respectively. The atoms defined as being in the bcc configuration are colored in dark gray and the other atoms were colored in white in the Figs. 3, 6 and 7. However, note that the determination of the bcc configuration at a high temperature near the melting point includes an inherent error due to the thermal vibration.

\section{Results and Discussion}

\subsection{Estimation of Kinetic Coefficients}

First, a kinetic coefficient was estimated from the propagation velocity of the planar solid-liquid interface as a function of temperature. A system containing planar solidliquid interfaces was prepared by connecting cells of solid bcc crystal and liquid by following procedures. Two bcc crystals with $20 \times 10 \times 10$ unit cells (4 000 atoms) with (100) plane appearing on the boundary, located with the longest side parallel to the $x$-axis, were prepared in the periodical cell. In a similar way, two bcc crystals with $17 \times 8 \times 12$ (6528 atoms) with (110) plane appearing on the boundary were prepared. Each crystal was annealed separately at $10 \mathrm{~K}$ and $3500 \mathrm{~K}$ for $100 \mathrm{ps}$ to obtain cells with the solid and the liquid structure. Then, one solid cell and five liquid cells were connected to create a solid-liquid coexisting system, with (100) and (110) plane appearing on the interfaces, respectively. Two interfaces appear in the system due to the periodic boundary condition. In order to remove the pressure and temperature difference between the liquid and solid, the system was then annealed under zero pressure for $200 \mathrm{ps}$ at the melting point, $2400 \mathrm{~K}$. For the purpose of checking for the size effects, systems with twice the size in $z$-direction of initial conditions prepared above were also made.

The solidification and melting behavior of the initial configuration were observed by annealing the system with temperatures around the melting point. The annealing temperature was maintained constant throughout the calculation, and they were varied from 2200 to $2450 \mathrm{~K}$ with the interval of $25 \mathrm{~K}$. Figure 1 shows snapshots of the solidification and melting at the planar solid-liquid interface at 2300 and $2450 \mathrm{~K}$, respectively For the temperatures above the reported melting point, $2400 \mathrm{~K}$, melting of a bcc crystal occurred at the interface, while the solidification at the interface was observed for the temperatures below the melting point. The propagation velocity of the solid-liquid interface appeared to be almost constant through the calculation time of $400 \mathrm{ps}$. Shape of the interface appeared to be planar regardless of the size of the initial condition. Figure 2 shows the interfacial velocity, $v$, as a function of the undercooling temperature, $\Delta T$. The linear relationships are

Table 1. Parameters of Finnis-Sinclair potential for iron-iron bond.

\begin{tabular}{|c|c|c|c|c|c|c|}
\hline$d[\AA]$ & $A[\mathrm{eV}]$ & $b$ & $c[\AA]$ & $c_{0}$ & $c_{1}$ & $c_{2}$ \\
\hline 3.569745 & 1.828905 & 1.8 & 3.4 & 1.2371147 & -0.3592185 & -0.0385607 \\
\hline
\end{tabular}


observed to hold fairly well over these temperatures, for both (100) and (110) planes. The correlation coefficient of the relationship is known to be the kinetic coefficient, $\mu$, as:

$$
v=\mu \Delta T
$$

The resulting values of $\mu$ were $30.5 \mathrm{~cm} / \mathrm{s} \mathrm{K}$ and $25.7 \mathrm{~cm} / \mathrm{s} \mathrm{K}$ for the (100) and (110) orientations, respectively. The kinetic coefficients obtained above are summarized with references calculated by Sun et al. ${ }^{13)}$ using embedded atom method $\left(\mathrm{EAM}(\mathrm{ABCH}),{ }^{32)} \operatorname{EAM}\left(\mathrm{MH}(\mathrm{SA})^{2}\right)^{33)}\right)$ and a pair potential in Table 2. The melting point, $T_{\mathrm{M}}$ and latent heat, $\Delta H$ of bcc iron by these interatomic potentials are also listed for reference. In all potentials, there is a same trend in anisotropy of the kinetic coefficients: $\mu_{(100)}>\mu_{(110)}$. The

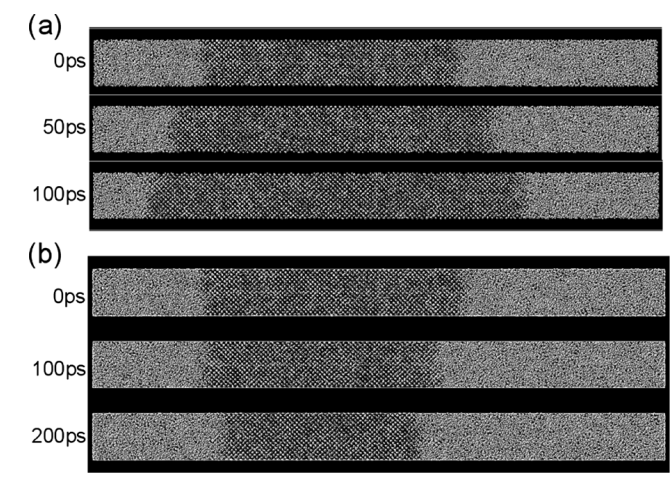

Fig. 1. Snapshot of the (a) solidification and (b) melting at the planar solid-liquid interface at 2300 and $2450 \mathrm{~K}$, respectively.

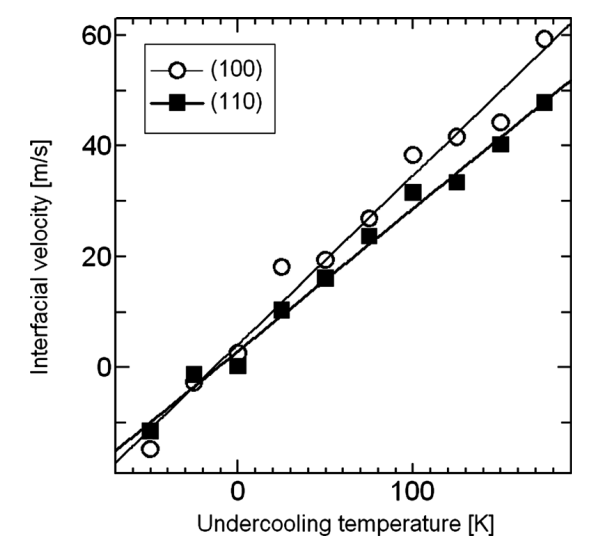

Fig. 2. Interfacial velocity as a function of undercooling temperature for (100) and (110) orientation. Positive values of interfacial velocity express solidification in the figure. ratio, $\mu_{(100)} / \mu_{(110)}$ was varied from 1.19 (FS potential) to $1.37\left(\mathrm{MH}(\mathrm{SA})^{2}\right.$ potential). It was confirmed that there is a negative correlation between the melting point and kinetic coefficient. That is, the kinetic coefficient estimated from the $\operatorname{EAM}\left(\mathrm{MH}(\mathrm{SA})^{2}\right)$ is higher than that from the other interatomic potentials, whereas melting point estimated from the $\operatorname{EAM}\left(\mathrm{MH}(\mathrm{SA})^{2}\right)$ is lower than those from the other interatomic potentials. By comparison, the latent heat correlates positively to the melting point.

\subsection{Growth and Shrink of Freestanding Spherical Crystals in Undercooled Liquid}

In order to investigate the curvature effect on thermodynamic and kinetic properties of the solid-liquid interface, the growing and shrinking behavior of spherical crystals embedded in the undercooled liquid was examined based on previous reports. ${ }^{21)}$ The initial condition was obtained by embedding a spherical crystal into a cell filled with the liquid iron as follows. Spherical crystals were prepared by cooling freestanding liquid droplets from 3000 to $0 \mathrm{~K}$ at a rate of $1 \mathrm{~K} / 5 \mathrm{ps}^{24)}$ The liquid cells were obtained by heating bcc crystals at $3500 \mathrm{~K}$ under zero pressure for $40 \mathrm{ps}$. Eighteen pairs of solid crystal and liquid cell were prepared with a number of atoms of $2 n^{3}$ and $16 n^{3}(n: 7-24)$, respectively. For each pair, the solid crystal was inserted into the liquid cell, while omitting all liquid atoms located within $2.5 \AA$ from a solid atom to avoid the unexpected proximity between liquid and solid atoms at the interface. Then, the system is quenched at $10 \mathrm{~K}$ for $200 \mathrm{ps}$ to fill the gaps between the solid crystal and liquid iron, which would make an initial condition. The radius of the spherical crystal was defined from the distribution of potential energy in the system. That is, there is a gap in the potential energy at the solid-liquid interface. In the initial configuration, the average of the potential energy per atom in bcc crystal and liquid iron were about -4.28 and $-4.12 \mathrm{eV} /$ atom, respectively. In this study, assuming solid crystal to be spherical, the radius of a sphere is defined as the average distance from a center of mass of solid crystal where potential energy crosses $-4.2 \mathrm{eV}$.

Each initial configuration prepared above was then annealed at various temperatures below the melting point. Figure 3 shows snapshots of cross-sectional view for two crystals with radius of 33.7 and $39.4 \AA$ annealed at $2290 \mathrm{~K}$. It was observed that the smaller crystal shrank due to the melting, whereas the bigger one grew as a result of solidification. Therefore, it can be expected that there is a critical

Table 2. Melting point, $T_{\mathrm{M}}$, latent heat, $\Delta H$, and kinetic coefficients, $\mu$, for (100) and (110) orientation for bcc iron estimated using Finnis-Sinclair potential and other interatomic potentials.

\begin{tabular}{|c|c|c|c|c|}
\hline \multirow{2}{*}{ Potential } & & \multirow{2}{*}{$\Delta H[\mathrm{ev} /$ atom $]$} & \multicolumn{2}{|c|}{$\mu[\mathrm{cm} / \mathrm{sK}]$} \\
\cline { 4 - 5 } & $T_{M}[\mathrm{~K}]$ & $\mathrm{bcc}(100)$ & $\mathrm{bcc}(110)$ \\
\hline Finnis-Sinclair & $2400 \pm 10^{[29]}$ & $0.22^{[24]}$ & 30.5 & 25.7 \\
\hline EAM $(\mathrm{ABCH})^{[32]}$ & $2358 \pm 4^{[13]}$ & $0.218^{[13]}$ & $32.5 \pm 1.6^{[13]}$ & $24.6 \pm 1.4^{[13]}$ \\
\hline EAM $\left(\mathrm{MH}(\mathrm{SA})^{2}\right)^{[33]}$ & $1772 \pm 2^{[13]}$ & $0.162^{[13]}$ & $45.8 \pm 3.8^{[13]}$ & $33.5 \pm 1.0^{[13]}$ \\
\hline Pair & $2311.8 \pm 7^{[13]}$ & $0.259^{[13]}$ & $30.8 \pm 7.0^{[13]}$ & $22.6 \pm 4.0^{[13]}$ \\
\hline Experimental & $1811.0^{[13]}$ & $0.143^{[13]}$ & - & - \\
\hline
\end{tabular}




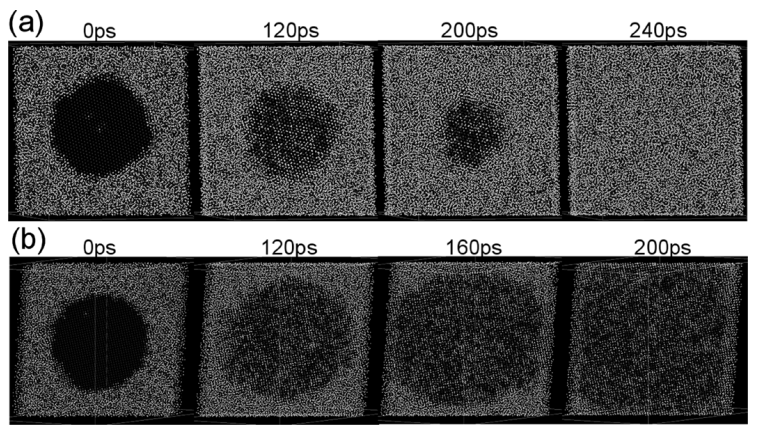

Fig. 3. Snapshots of cross-sectional view of calculation cell containing freestanding spherical crystal of (a) 33.7 and (b) $39.4 \AA$ in the liquid iron at $2290 \mathrm{~K}$. The dark gray and white circles represent atoms with and without the bcc configuration, respectively.

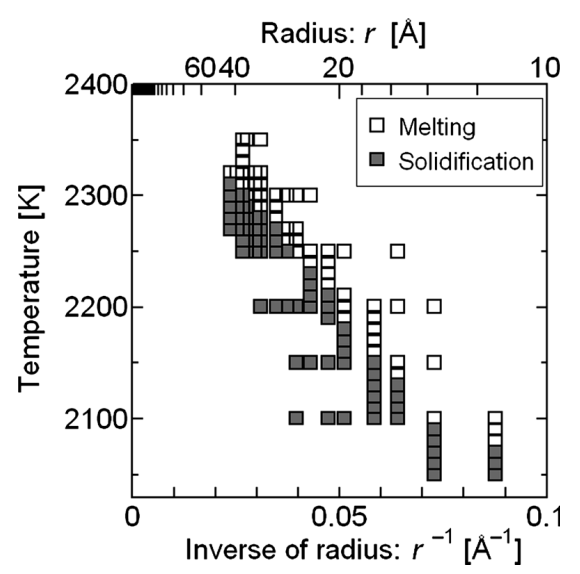

Fig. 4. Matrix showing the direction of solidification or melting as a function of temperature and the inverse of the radius for freestanding spherical crystal. White and gray squares represent the melting and solidification, respectively.

radius, which divides growing and shrinking of a crystal at this temperature. Figure $\mathbf{4}$ shows a matrix showing the direction of solidification or melting as a function of temperature and the inverse of the radius. It was confirmed that there is no condition that allows the spherical crystal to exist stably. That is, the crystal in the undercooled liquid shrank or grew in all condition. However, the critical radius can be defined from a bifurcation radius between the largest melting radius and the smallest solidification radius, since the trend changes monotonically from melting to solidification as increasing crystal radius at every temperature. In other words, at constant radius, there is a critical temperature, which divides melting and solidification of the crystal in the undercooled liquid. Hence, the critical temperature for each initial condition can be defined to be the average temperature between the lowest melting temperature and the highest solidification temperature. In this study, the critical temperature can be estimated within the error of $\pm 5 \mathrm{~K}$ since the annealing temperature was changed with a $10 \mathrm{~K}$ interval around the critical temperature. The critical temperature is negatively correlated with inverse of radius, which agrees with depression of melting point of freestanding nanoparticles as a function of particle radius. ${ }^{23,24)}$

The critical temperature was summarized as the undercooling temperature from the bulk melting point, $2400 \mathrm{~K}$ in Fig. 5 (black dots). The critical temperature for the semi-

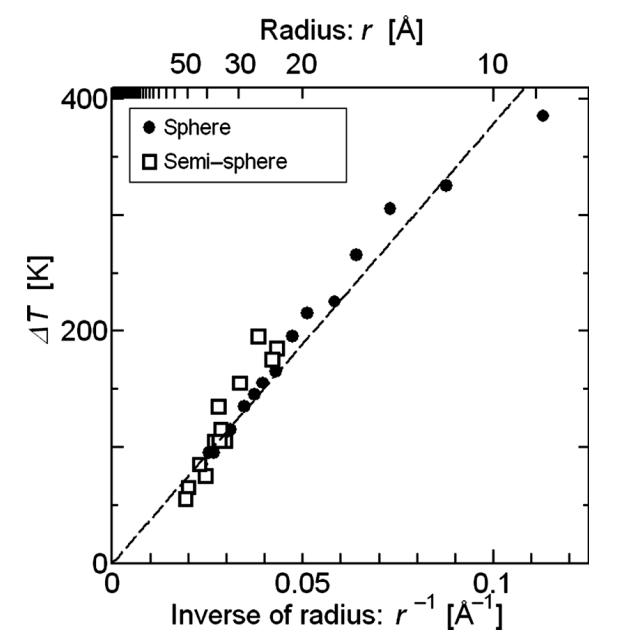

Fig. 5. Depression of the critical temperature from the bulk melting point as a function of the inverse of crystal radius. Black dots and white squares represent critical temperature of the freestanding spherical crystal and the semi-spherical crystal on the substrate, respectively.

spherical crystal is also plotted (white squares), which will be discussed in Sec. 3.3. It was confirmed that depression of the critical temperature from the bulk melting point was proportional to the inverse of the critical radius, which is in agreement with the classical theory of homogeneous nucleation. ${ }^{34-36)}$ According to the classical nucleation theory, a spherical crystal embedded in the undercooled melt causes a difference in free energy, $\Delta G$, expressed in terms of particle radius $r$ :

$$
\begin{gathered}
\Delta G=4 \pi r^{2} \sigma_{\mathrm{SL}}-\frac{4 \pi r^{3}}{3} \Delta G_{\mathrm{V}} \\
\Delta G_{\mathrm{V}}=\frac{\Delta T \Delta H}{T_{\mathrm{M}}} \ldots \ldots \ldots \ldots
\end{gathered}
$$

where $\Delta G_{\mathrm{V}}$ expresses the Gibbs free energy difference between the liquid and solid per unit volume, and $\sigma_{\mathrm{SL}}$ is the solid-liquid interfacial energy. The critical radius, $r^{*}$ and critical activation energy, $\Delta G^{*}$ are derived by differentiation of the Eq. (6) and setting differentiation to be zero as,

$$
\begin{aligned}
& \frac{d \Delta G}{d r}=8 \pi r \sigma_{\mathrm{SL}}-4 \pi r^{2} \Delta G_{\mathrm{V}}=0, \quad \text { at } r=r^{*} \\
& r^{*}=\frac{2 \sigma_{\mathrm{SL}}}{\Delta G_{\mathrm{V}}}=\frac{2 \sigma_{\mathrm{SL}} T_{\mathrm{M}}}{\Delta H} \frac{1}{\Delta T} \\
& \Delta G^{*}=\frac{16}{3} \frac{\pi \sigma_{\mathrm{SL}}^{3}}{\Delta G_{\mathrm{V}}^{2}}
\end{aligned}
$$

Hence,

$$
\Delta T=\frac{2 \sigma_{\mathrm{SL}} T_{\mathrm{M}}}{\Delta H} \frac{1}{r^{*}}
$$

As shown in Fig. 5, depression of the critical temperature from the bulk melting point was proportional to the inverse of the critical radius. Hence, the solid-liquid interfacial energy can be estimated from Eq. (11) by using the propor- 
tional constant of the fitted line in Fig. 5. The solid-liquid interfacial energy was actually estimated in Sec. 3.4 after examining the growing and shrinking behavior of semispherical crystals on the substrate.

\subsection{Growth and Shrink of Semi-spherical Crystals on the Substrate Contacting Undercooled Liquid}

In Sec. 3.2, the correlation between critical temperature and the inverse of the critical radius was discussed using a spherical crystal as a homogeneous nucleus in the classical nucleation theory. However, considering experimental conditions, nucleation from a wall of a container is known to be favorable than homogeneous nucleation as a result of decreased activation energy for nucleation, which is known as heterogeneous nucleation. In this section, the growing and shrinking behavior of semi-spherical crystals supported on the substrate contacting in the undercooled liquid was examined and the results are compared with the case of the spherical crystals. In order to express an effect of the substrate, interactions between the bottom boundary of the cell and iron atoms are approximated by the one-dimensionally averaged Lennard-Jones (1DLJ) potential. The 1DLJ potential is expressed in terms of a distance from a boundary, $z$ :

$$
F(z)=\varepsilon\left\{\frac{1}{5}\left(\frac{z}{\sigma}\right)^{-10}-\frac{1}{2}\left(\frac{z}{\sigma}\right)^{-4}\right\} \ldots \ldots \ldots . . .
$$

The 1DLJ potential represents the integration of the interaction between an iron atom in the cell and the substrate, which is described by a standard 12-6 Lennard-Jones (LJ) potential function. Deviation of the 1DLJ potential from a LJ potential was summarized in Ref. 37). The parameter $\sigma$ is to be $3.37 \AA$. The integrated depth of the 1DLJ potential, $\varepsilon$, was varied from 1.0 to $3.0 \mathrm{eV}$ at intervals of $0.5 \mathrm{eV}$ to describe the variable affinity between the atoms and the substrate.

A semi-spherical crystal was obtained by taking similar steps to the spherical crystal. The initial semi-spherical crystal was obtained by cooling a liquid droplet on a substrate with interaction parameters of $2.0 \mathrm{eV}$ at a rate of $1 \mathrm{~K} / 5 \mathrm{ps.}^{25)}$ Number of solid atoms was varied from 4394 to 6750 atoms $\left(2 n^{3}: n=13-15\right)$. The liquid cell was prepared by heating the bcc crystal in the periodical cell under zero pressure at $3500 \mathrm{~K}$. The number of liquid atoms remains constant at $109744\left(16 n^{3}: n=19\right)$, which is projected to be large enough for each condition. Solid atoms are then inserted into a liquid cell, with various $\varepsilon$ values noted above, while omitting all liquid atoms located within $2.5 \AA$ from a solid atom in the same way as the spherical crystals above. The height of the cell for main calculation was set to be twice the height of solid-liquid coexisting system, leaving top of the system open to vacuum since the mirror boundary was applied at the top and bottom boundary to treat the bottom boundary as the substrate. The solid-vacuum-liquid coexisting system is then annealed at the melting temperature of bulk, $2400 \mathrm{~K}$ for $40 \mathrm{ps}$ to obtain various shapes of the semi-spherical crystal on the substrate with various interaction energies. For the purpose of measuring radius, the system is then quenched at $10 \mathrm{~K}$ for $40 \mathrm{ps}$. Figure 6(a) shows the initial configurations obtained by above process with various interactions, $\varepsilon$, which is started from the solid particle with 6750 atoms. It was observed that the contact angle became small with increasing the interaction energy. The radius of the semi-sphere was estimated from the height of the droplet, $x_{1}$ and the radius of the cross-sectional circle at the bottom of the crystal, $x_{2}$ (see Fig. 6(b)) on the assumption that the semi-spherical crystal on the substrate was a part of sphere:

$$
r=\frac{x_{1}^{2}+x_{2}^{2}}{2 x_{1}}
$$

From the coordinates, $x_{1}$ and $x_{2}$, the height of the center of circle, $h$ and contact angle, $\theta$ can be defined as ${ }^{37)}$ :

$$
\begin{array}{r}
h=\frac{x_{1}^{2}-x_{2}^{2}}{2 x_{1}} \ldots \ldots \ldots . . . . . \\
\cos \theta=-\frac{h}{R}=\frac{x_{2}^{2}-x_{1}^{2}}{x_{2}^{2}+x_{1}^{2}}
\end{array}
$$

These coordinates, $x_{1}$ and $x_{2}$ were measured with potential energy distribution similar to the Sec. 3.2. The average potential energy at the solid-liquid interface was again set to be $-4.2 \mathrm{eV} /$ atom.

The initial coordination prepared above was annealed at various temperatures below the melting point. The pressure was not controlled during main calculation since the vacuum region enables the excess pressure to evacuate easily. Figure 7 shows growth and melting of a semi-spherical crystal prepared by solid particle with 6750 atoms, one
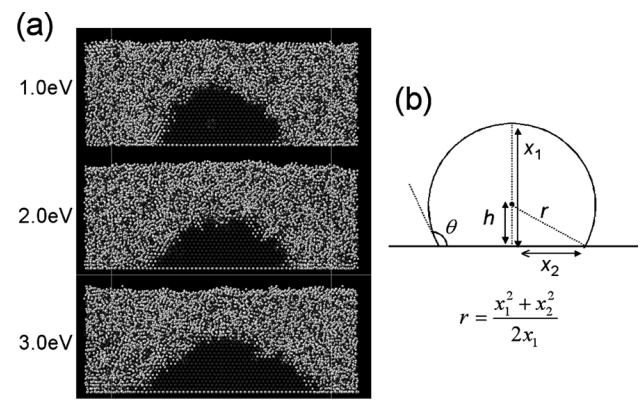

Fig. 6. (a) The initial configurations for main calculation with semi-spherical crystal on the substrate of various interaction energy. (b) Definition of the radius, $r$, height, $h$ and contact angle $\theta$, of the semi-spare crystal on the substrate.

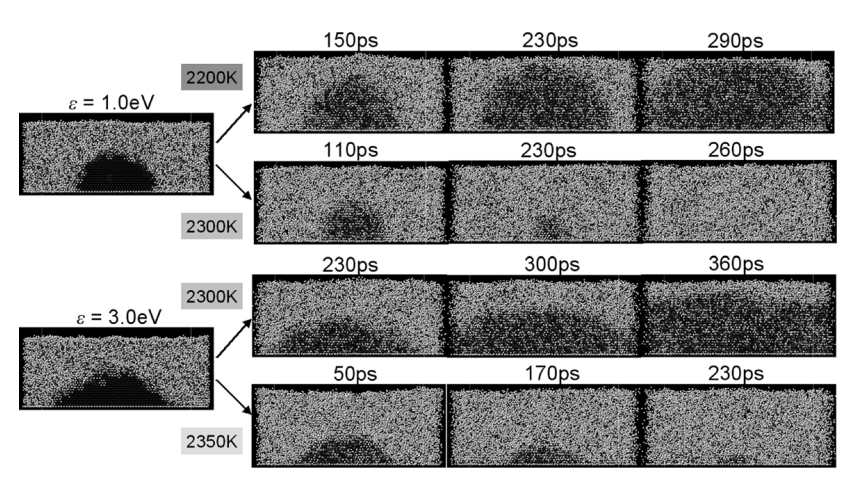

Fig. 7. Snapshots of the growing and shrinking process of the semi-spherical crystal on the substrate of weaker $(\varepsilon=1.0 \mathrm{eV})$ and stronger interaction $(\varepsilon=3.0 \mathrm{eV})$. 
with a weaker interaction $(\varepsilon=1.0 \mathrm{eV})$ between the substrate and atoms, and the other with a stronger interaction $(\varepsilon=3.0 \mathrm{eV})$ at various temperatures. The semi-spherical crystal on the substrate shrank due to the melting at higher temperature $(2300 \mathrm{~K}$ for $\varepsilon=1.0 \mathrm{eV}$ and $2350 \mathrm{~K}$ for $\varepsilon=$ $3.0 \mathrm{eV}$ ), whereas the one grew as a result of solidification at lower temperature $(2200 \mathrm{~K}$ for $\varepsilon=1.0 \mathrm{eV}$ and $2300 \mathrm{~K}$ for $\varepsilon=3.0 \mathrm{eV}$ ) in the case of both weaker and stronger interactions. Therefore, there is also a critical temperature, which divides melting and solidification as in the case of the spherical crystal. The critical temperature seems higher (i.e., the lower undercooling temperature) for the case of the stronger interaction. That is, the lower contact angle due to the strong interaction between metal atoms and substrate resulted in the lower undercooling temperature, which is reasonable from the point of view of experimental knowledge. ${ }^{1)}$ Moreover, it is interesting to see that the contact angle of the semi-spherical crystal remains almost constant during solidification and melting. Thus the idea of balances of the surface tensions of solid-liquid, liquid-substrate and solid-substrate interface at the triple point in the Young's equation seems to be valid in the range of the interaction energy, $\varepsilon$ used in this study.

Figure 8 shows the matrix showing the direction of solidification or melting as a function of temperature and the inverse of the radius. As in the case of the spherical crystal, the solidification and melting occur above and below the critical temperature, respectively. The critical temperature was estimated within the error of $\pm 5 \mathrm{~K}$ from data with a $10 \mathrm{~K}$ interval around the critical temperature. The estimated critical temperature is again negatively correlated with the inverse of radius. For comparison, the critical temperature estimated from the semi-spherical crystal on the substrate was plotted in the Fig. 5 (as white squares) where the critical temperature from the freestanding spherical crystal was summarized as a function of the inverse of crystal radius. It was confirmed that the depression of critical temperature from the semi-spherical crystal on the substrate was also proportional to the inverse of crystal radius and the proportional constant agrees well with that from the freestanding spherical crystal. It is reasonable considering

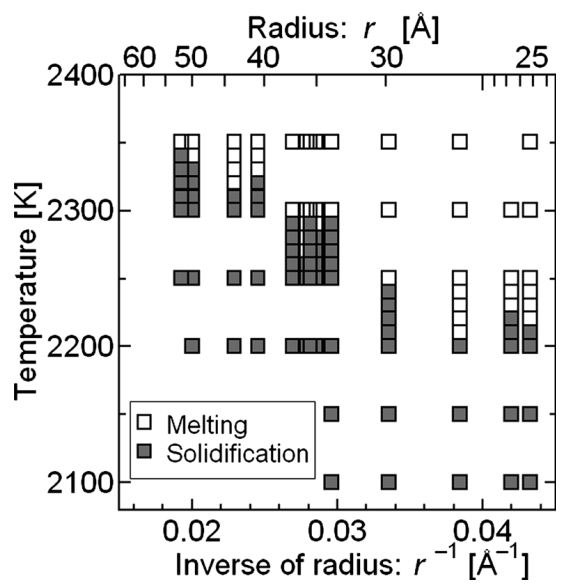

Fig. 8. Matrix showing the direction of solidification or melting as a function of temperature and the inverse of the radius for semi-spherical crystal on the substrate. White and gray squares represent the melting and solidification, respectively. the classical nucleation theory for heterogeneous nucleation. That is, the activation energy for the heterogeneous nucleation, $\Delta G^{*}{ }_{\text {hetero }}$ changes as a function of the contact angle as ${ }^{1)}$.

$$
\Delta G_{\text {hetero }}^{*}=\Delta G^{*} \frac{\left(2-3 \cos \theta+\cos ^{3} \theta\right)}{4}
$$

However, the critical radius for heterogeneous nucleation is expressed as same as in Eq. (9). Hence, there is a same trend in depression of the critical temperature as a function of the inverse of the melting point for both freestanding spherical crystal and semi-spherical crystal on the substrate.

\subsection{Estimation of Solid-Liquid Interfacial Energy}

In general, the capillary effect has been widely investigated in the study of solidification. It is well known that equilibrium temperature of the solid-liquid interface decreases during solidification due to the curvature of the interface. ${ }^{1)}$ This is called the Gibbs-Thomson effect and modeled as

$$
\Delta T=\Gamma K, \quad K=\frac{1}{r_{1}}+\frac{1}{r_{2}}
$$

where $\Gamma$ is the Gibbs-Thomson coefficient, $K$ is the curvature, and $r_{1}$ and $r_{2}$ are the principal radii of curvature. In the case of the curvature of a sphere, the Gibbs-Thomson Eq. (17) corresponds to the Eq. (11). Hence, the Gibbs-Thomson coefficient and the solid-liquid interface of the bcc iron can be estimated using the proportional coefficient of the fitted line in Fig. 5. The slope of the fitted line in Fig. 5 gives an estimated value of the Gibbs-Thomson coefficient to be $1.9 \times 10^{-7} \mathrm{~m} \mathrm{~K}$, which is equivalent to the experimental value of iron measured by Turnbull. ${ }^{5)}$ From the Gibbs-Thomson equations, the solid-liquid interfacial energy can be estimated using the latent heat and melting point of the bulk system, listed in Table 2, to be $1.7 \times 10^{-1} \mathrm{~J} / \mathrm{m}^{2}$, which is about $20 \%$ lower than the experimental value of $2.04 \times 10^{-1} \mathrm{~J} / \mathrm{m}^{2}$. ${ }^{5)}$ The solid-liquid interface of bcc iron estimated from Gibbs-Thomson coefficient using FS potential and that estimated from capillary fluctuation method calculated by Sun et al. ${ }^{13)}$ are summarized in Table 3. Actually, it is difficult to find good correlation between the solid-liquid interface and melting point (or latent heat) among the results listed in the table. Hence, we note that we have to be careful to interpret these results from a quantitative point of view since it is related to the feature of

Table 3. Solid-liquid interfacial energy estimated using Finnis-Sinclair and other interatomic potentials.

\begin{tabular}{|c|c|}
\hline Potential & $\sigma_{\mathrm{SL}}\left[\mathrm{mJ} / \mathrm{m}^{2}\right]$ \\
\hline Finnis-Sinclair & 170 \\
\hline EAM $(\mathrm{ABCH})$ & $206 \pm 10^{[13]}$ \\
\hline EAM (MH(SA) $\left.)^{2}\right)$ & $175 \pm 11^{[13]}$ \\
\hline Pair & $221 \pm 14^{[13]}$ \\
\hline Experimental & $204^{[5]}$ \\
\hline
\end{tabular}


the interatomic potentials.

\section{Conclusion}

By performing classical MD simulations, the thermodynamic and kinetic properties of solid-liquid interface of bcc iron have been investigated. The kinetic coefficients for the (100) and (110) orientations were estimated from propagation velocity of the planar solid-liquid interface. The anisotropy of kinetic coefficients, $\mu$, was confirmed to be $\mu_{(100)}>\mu_{(110)}$, which is similar to previous reports using other interatomic potentials. Then, growth and melting behavior of the freestanding spherical crystal and semispherical crystal on the substrate in the undercooled liquid was calculated. It was found that there is a critical temperature dividing shrinking or growing of both the freestanding spherical crystal and semi-spherical crystal on the substrate. The depression of the critical temperature was proportional to the inverse of crystal radius both in the case of the spherical and semi-spherical crystal. Then, the solidliquid interfacial energy was estimated from the proportional coefficient of critical temperature as a function of the inverse of crystal radius.

Since the estimation method of the solid-liquid interfacial energy has not been established, it is significant to reveal in this study that the solid-liquid interfacial energy of bcc iron estimated from Gibbs-Thomson relation shows a proper value compared with interfacial energies from other methods such as the cleaving technique and capillary fluctuation method. It is promising to apply this methodology to the estimation of the solid-liquid interfacial energy of other metals and alloy, which gives much information from the atomic point of view.

\section{Acknowledgement}

Part of this work was financially supported by Grantin-Aid for Young Scientists (a) (No. 21686021) from MEXT, Japan, Grant-in-Aid for Exploratory Research (No. 20656036) from JSPS, Japan, and the LISM (Layer-Integrated Steels and Metals) project by MEXT, Japan.

\section{REFERENCES}

1) W. Kurz and D. J. Fisher: Fundamentals of Solidification 4th revised edition, Trans Tech Publication, Aedermannsdorf, (1998), 21.

2) W. J. Briels and H. L. Tepper: Phys. Rev. Lett., 79 (1007), 5074.

3) M. E. Glicksman and R. J. Shaefer: J. Cryst. Growth, 1 (1967), 297.

4) G. H. Rodway and J. D. Hunt: J. Cryst. Growth, 112 (1991), 554.

5) D. Turnbull: J. Appl. Phys., 21 (1950), 1022.

6) D. Turnbull and R. E. Cech: J. Appl. Phys., 21 (1950), 804.

7) M. Gündüz and J. D. Hunt: Acta Metall., 33 (1985), 1651.

8) M. Erol, N. Maraşlı, K. Keslioğlu and M. Gündüz: Scr. Mater, 51 (2004) 131.

9) J. Q. Broughton, G. H. Gilmer and K. A. Jackson: Phys. Rev. Lett., 49 (1993), 1496.

10) E. Burke, J. Q. Broughton and G. H. Gilmer: J. Chem. Phys., 89 (1988), 1030.

11) M. Amini and B. B. Laird: Phys. Rev. Lett., 97 (2006), 216102.

12) J. J. Hoyt, B. Sadigh, M. Asta and S. M. Foiles: Acta Mater, 47 (1999), 3181.

13) D. Y. Sun, M. Asta and J. J. Hoyt: Phys. Rev. B, 69 (2004), 174103.

14) J. Q. Broughton and G. H. Gilmer: J. Chem. Phys., 84 (1986), 5759.

15) R. L. Davidchack and B. B. Laird: Phys. Rev. Lett., 85 (2000), 4751.

16) R. L. Davidchack and B. B. Laird: J. Chem. Phys., 118 (2003), 7651

17) J. J. Hoyt, M. Asta and A. Karma: Phys. Rev. Lett., 86 (2001), 5530.

18) J. R. Morrs and X. Song: J. Chem. Phys., 119 (2003), 3920.

19) X.-M. Bai and M. Li: J. Chem. Phys., 112 (2005), 224510.

20) X.-M. Bai and M. Li: J. Chem. Phys., 124 (2006), 124707.

21) Y. Shibuta, Y. Watanabe and T. Suzuki: Chem. Phys. Lett., 475 (2009), 264

22) M. W. Finnis and J. E. Sinclair: Philos. Mag. A, 50 (1984), 45.

23) Y. Shibuta and T. Suzuki: Chem. Phys. Lett., 445 (2007), 265.

24) Y. Shibuta and T. Suzuki: J. Chem. Phys., 129 (2008), 144102.

25) Y. Shibuta and T. Suzuki: Chem. Phys. Lett., 486 (2010), 137.

26) S. Tateyama, Y. Shibuta and T. Suzuki: Scr. Mater, 59 (2008), 971.

27) S. Tateyama, Y. Shibuta and T. Suzuki: ISIJ Int., 50 (2010), 1211.

28) M. W. Finnis and J. E. Sinclair: Philos. Mag. A, 53 (1986), 161.

29) Y. Shibuta, S. Takamoto and T. Suzuki: ISIJ Int., 48 (2008), 1582.

30) H. J. C. Berendsen, J. P. M. Postma, W. F. van Gunsteren, A. DiNola and J. R. Haak: J. Chem. Phys., 81 (1984), 3684.

31) H. C. Andersen: J. Chem. Phys., 72 (1980), 2384.

32) G. J. Ackland, D. J. Bacon, A. F. Calder and T. Harry: Philos. Mag. A, 75 (1997), 713.

33) M. I. Mendelev, S. Han, D. J. Srolovitz, G. J. Ackland, D. Y. Sun and M. Asta: Philos. Mag., 83 (2003), 3977.

34) D. Turnbull and J. C. Fisher: J. Chem. Phys., 17 (1949), 71.

35) D. T. Wu: Solid State Phys., 50 (1997), 37.

36) R. W. Balluffi, S. M. Allen and W. C. Carter: Kinetics of Materials, Wiley, Hoboken, (2005), 460.

37) Y. Shibuta and T. Suzuki: Phys. Chem. Chem. Phys., 12 (2010), 731. 